\title{
Diagnostic accuracy of low-dose dual- source cardiac computed tomography as compared to surgery in univentricular heart patients
}

\author{
Narumol Chaosuwannakit ${ }^{*}$ (D) and Pattarapong Makarawate ${ }^{2}$
}

\begin{abstract}
Background: To evaluate the ability of low radiation dose dual-source computed tomography (DSCT) to depict the features of morphological univentricular heart and to define accuracy by comparing findings with surgery.

Methods: Low radiation dose dual-source cardiac computed tomography (CCT) of 33 cases of functional univentricular heart preliminary diagnosis by echocardiography compared with the results of surgery were retrospectively analyzed (aged 1 day to 4 years, median 5 months). The appropriate dose reduction strategies and iterative reconstruction were applied.

Results: Thirty three univentricular heart patients were classified into three types according to Anderson's classification method, including 16 cases (48.5\%) univentricular of right ventricular type with rudimentary chamber of left ventricle, 11 cases (33.3\%) univentricular of left ventricular type with rudimentary chamber of right ventricle and 6 cases (18.2\%) univentricular heart of indeterminate type without rudimentary chamber. The extracardiac malformation such as hypoplastic aortic arch, coronary artery fistula, total anomalous pulmonary venous returns or hypoplastic lung were presented frequently. The overall sensitivity and specification of cardiac CT was 100\% compared to the results of surgery. The procedural dose-length product was $18 \pm 5 \mathrm{mGy}-\mathrm{cm}$, and unadjusted and adjusted radiation doses were 0.25 and $0.64 \mathrm{mSv}$, respectively.

Conclusion: Cardiac CT can diagnose accurately and be performed with a low radiation exposure in patients with the functional univentricular heart disease. The aorta, pulmonary artery and lung can be evaluated completely and simultaneously as well. Cardiac $C T$ is an effective advanced non-invasive imaging modality to comprehensive evaluation the functional univentricular heart patients, particularly if cardiac MRI poses a high risk or is contraindicated.
\end{abstract}

Keywords: Univentricular heart, Single ventricle congenital heart disease, Cardiac $C T$, Dual-source $C T$

\section{Background}

Functional univentricular heart disease is a spectrum of severe congenital heart disease, with multiple anatomic variations but similar surgical treatment strategies. V Functional univentricular heart disease is anatomically defined as (1) connection of both atria to the same ventricle (2:1 connection) or as (2) connection of both to atria separate ventricles, one of which is hypoplastic (1:1 connection) $[1,2]$.

\footnotetext{
*Correspondence: narumol_chao@yahoo.com

${ }^{1}$ Radiology Department, Faculty of Medicine, Khon Kaen University, Khon Kaen 40000, Thailand

Full list of author information is available at the end of the article
}

With the advent of advanced palliative and corrective surgical procedures, Functional univentricular heart disease patients are living longer into adulthood compared to two or three decades ago [3], and they are more frequently undergoing imaging to assist in clinical and surgical management. However, interpreting an imaging examination of a functional univentricular heart disease patient is a daunting task, not only because of unusual anatomy and varied post operative appearances but also because of the rarity of the functional univentricular heart disease that makes it difficult for the imaging specialist to maintain diagnostic proficiency.

(C) The Author(s). 2018 Open Access This article is distributed under the terms of the Creative Commons Attribution 4.0 International License (http://creativecommons.org/licenses/by/4.0/), which permits unrestricted use, distribution, and 
Echocardiography and cardiac magnetic resonance imaging (CMR) are the main imaging modalities used in adult patients with complex congenital heart disease. However, cardiac CT offers complementary imaging and can be performed safely in unwell patients with single ventricle physiology. Echocardiography is insufficient for evaluation of the thoracic vasculature or for reproducible estimation of ventricular function [4, 5]. Cardiac MRI (CMR) is commonly performed for these indications, but it requires relatively long imaging times, deep sedation, or anesthesia in young children. Many older patients have metallic implants with an artifact that degrades magnetic resonance imaging (MRI) quality [6]. In addition, it is relatively contraindicated in those with pacemakers and defibrillators as these devices have been known to cause an imaging artifact [7]. Recent findings of cerebral gadolinium deposits suggest that MRI use be carefully considered when angiography is needed [8-10]. Cardiac CT has been shown to be accurate for the evaluation of anatomy and function for most indications of congenital heart disease (CHD), [11] but there has not been a report on image quality, nor has a correlation been made with interventional findings, in a cohort of single-ventricle patients across all stages of palliation.

\section{Methods}

\section{Patient population}

This retrospective study included 33 functional univentricular heart disease patients who underwent both cardiac CT and surgery, between February 2013-December 2016. The indications for cardiac CT were pre-operative evaluation of complex congenital heart and functional univentricular heart disease patients which is considered appropriate indications for cardiac CT, based on the expert consensus document of the Society of Cardiovascular computed tomography [11]. Exclusion criteria for cardiac CT included the presence of renal failure, and a history of allergic reaction to iodine-containing contrast agents. The present study was approved by the Ethics Committee of the Faculty of Medicine, Khon Kaen University, Khon Kaen, Thailand, and informed consent was obtained from all patients The IRB protocol number of this study is 800001189 .

\section{Dual-source cardiac CT (CCT) scanning protocol}

No sedation versus sedation with free breathing versus intubation, IV site and gauge, and adverse procedural events were determined from patient records. Nearly all patients could be performed cardiac CT with limited or no anaesthesia and with quiet respiration. Imaging was performed by using a second-generation dual-source CT scanner (Somatom Definition; Siemens Healthcare, Forchheim, Germany) with temporal resolution $=75 \mathrm{~ms}$. The radiation dose is kept to minimum by reducing the kilovoltage and tube current appropriately. For children weighing less than $10 \mathrm{~kg}, 10-19 \mathrm{~kg}$ and $20-30 \mathrm{~kg}$, we use $80 \mathrm{kV}$ and 80
mAs, $80 \mathrm{kV}$ and $100 \mathrm{mAs}$, and $100 \mathrm{kV}$ and $120 \mathrm{mAs}$, respectively. Other cardiac CT parameters were as follows: number of X-ray tubes, two; collimation, 128 detector rows of $0.5 \mathrm{~mm}$ each, with double sampling by using rapid alteration of the focal spot in the longitudinal direction ( $z$ flying focal spot). Prior to scanning, the pitch was set automatically by the scanner software. Depending on heart rate, pitch was set between 0.2 and 0.43 . Automated dose regulation methods such as CARE dose 4D (Siemens Healthcare) may be used to reduce the radiation. A bolus of iodinated contrast material $(350 \mathrm{mg} / \mathrm{mL}$, Omnipaque; GE Healthcare) at a dose of $1.5 \mathrm{ml} / \mathrm{kg}$ with dual-head power injector at a rate of $1.5-2.0 \mathrm{ml} / \mathrm{s}$ for a 22 -gauge cannula, $3.0 \mathrm{ml} / \mathrm{s}$ for a 20 - gauge cannula and $4.0-5.0 \mathrm{ml} / \mathrm{s}$ for a 18-gauge cannula followed by a $10-20 \mathrm{ml}$ of saline flush at a same rate to that of the contrast injection. For timing purposes, an automated bolus-tracking software was used, starting the scan automatically $6 \mathrm{~s}$ after contrast agent density in the descending aorta reached a predefined threshold of $130 \mathrm{HU}$. The entire volume of the heart and pulmonary arteries was covered during one breath-hold in approximately $5 \mathrm{~s}$ with simultaneous recording of the ECG trace. Patients were scanned in the supine position. Cardiac CT is performed from the thoracic inlet level to L1-L2. When there is suspicion of anomalous pulmonary venous drainage, the scan can be extended down to the lower border of the liver.

\section{Cardiac CT image analysis}

Cardiac CT image analysis was performed by two cardiovascular and thoracic radiologists in consensus (with a respective 11 and 10 years of experience in examining cardiovascular and thoracic CT scans) and blinded to the clinical data and the results of surgery. First, axial all image data are evaluated using a 3D post processing workstation with Syngo software (Siemens Healthcare). Various image reformatting techniques including curved planar reconstruction, maximum intensity projection (MIP), minimum intensity projection, and volume-rendering technique (VRT) are used to get all the clinically relevant information. Curved planar reformatting and MIP are primarily used to evaluate curved structures such as the pulmonary arteries and major aortopulmonary collateral arteries (MAPCAs). Minimum intensity projection is used to evaluate the airway and lung parenchyma. For 3D reformatting of the complex anatomy, VRT is used. Thin-section multiplanar reformatting is used for quantitative analysis of the structure in question.

\section{Cardiac surgery result analysis}

Cardiac surgery result analysis was performed by an experienced cardiologist blinded to cardiac $\mathrm{CT}$ results by retrospectively reviewed operative notes. 


\section{Radiation dose parameters}

The scanner platform, contrast, imaging sequence, CT dose-volume index, milligray (mGy), scan dose-length product (mGy-cm, $16 \mathrm{~cm}$ phantom), scan length, tube potential, and tube current were recorded for each scan. Individual scan and cumulative procedural dose-length products in $\mathrm{mGy}-\mathrm{cm}$ were recorded.

\section{Radiation dose estimation}

Procedural dose-length product was used to estimate the radiation dose. An unadjusted radiation dose in milliSievert $(\mathrm{mSv})$ was calculated by multiplying the dose- length product with the standard chest conversion factor given as scan dose-length product $\times 0.014$ [12] For patients $<18$ years of age, conversion factors were further calculated by age as follows: 0.039 for $\leq 0.50$ years; 0.026 for $0.51-2.50$ years; 0.018 for $2.51-7.50$ years; and 0.014 for patients $>7.50$ years $[13,14]$.

\section{Statistical analysis}

Continuous data were expressed as mean \pm SD. Statistical analyses were performed using SPSS software version 16 (SPSS, Inc., Chicago, IL, USA). A significance level of $p<0.05$ was considered a statistically significant result and all reported $p$-values were two-sided. Means were compared using unpaired t-test, and Mann-Whitney rank sum was used when data was not normally distributed.

\section{Results}

Thirty-three patients (20 boys, 13 girls; aged 1 day to 4 years, median 5 months) were included in this study over a period of 44 months. The mean time interval between cardiac CT and surgery was $1 \pm 10$ months (range, 3 days -14 months) and there were no clinical events between the 2 studies in any patient. Patient demographic data and cardiac CT scan information are lists as Table 1.

In patient analysis, out of 33 cardiac CT examinations, none of them was of non-diagnostic image quality. Thirty three univentricular heart patients were classified into three types according to Anderson's classification method, including 16 cases (48.5\%) univentricular of right ventricular type with rudimentary chamber of left ventricle (Fig. 1), 11 cases (33.3\%) univentricular of left ventricular type with rudimentary chamber of right ventricle (Fig. 2) and 6 cases (18.2\%) univentricular heart of indeterminate type without rudimentary chamber.

Of the 33 patients, significant unexpected findings were two hypoplastic aortic arch (Fig. 3), three coronary artery fistula (Fig. 2), ten pulmonary atresia, one infracardiac type total anomalous pulmonary venous returns (Fig. 4) and one hypoplastic left lung were presented. Ten patients were scanned without sedation, 22 patients were scanned with minimal to moderate sedation, and one patient was intubated during the scan. The patient who was intubated
Table 1 Patient demographic data and cardiac CT information

\begin{tabular}{ll}
\hline Characteristic & Value \\
\hline Age at scan (months), mean \pm SD (range) & $5 \pm 8(0-48)$ \\
Men, $n(\%)$ & $20(60.6 \%)$ \\
Height (cm), mean \pm SD & $90.8 \pm 23$ \\
Weight (kg), mean \pm SD & $3 \pm 10$ \\
Patient sedation & \\
• No sedation, $n(\%)$ & $10(30.3)$ \\
• Sedation with free breathing, $n(\%)$ & $22(66.6)$ \\
• Intubation, $n$ (\%) & $1(3.1)$ \\
Dose-length product (mGy-cm), mean \pm SD (range) & $18 \pm 5(15-21)$ \\
Unadjusted radiation dose (milliSievert), mean (range) & $0.25(0.21-0.29)$ \\
Adjusted radiation dose (milliSievert), mean (range) & $0.64(0.49-0.82)$ \\
\hline
\end{tabular}

for general anesthesia for multiple concurrent procedures including brain $\mathrm{CT}$ and cardiac $\mathrm{CT}$.

The procedural dose-length product was $18 \pm 5$ (15-21) $\mathrm{mGy}-\mathrm{cm}$, and unadjusted and adjusted radiation doses were $0.25(0.21-0.29)$ and $0.64 \quad(0.49-0.82) \mathrm{mSv}$, respectively.

All patients underwent subsequent staged palliation. No patient had additional advanced diagnostic studies before initial surgical palliation. No discrepancy was found of opinion regarding the classification of univentricular heart disease at time of surgery compared to cardiac CT findings. The overall sensitivity and specification of CTA was $100 \%$ compared to the results of surgery.

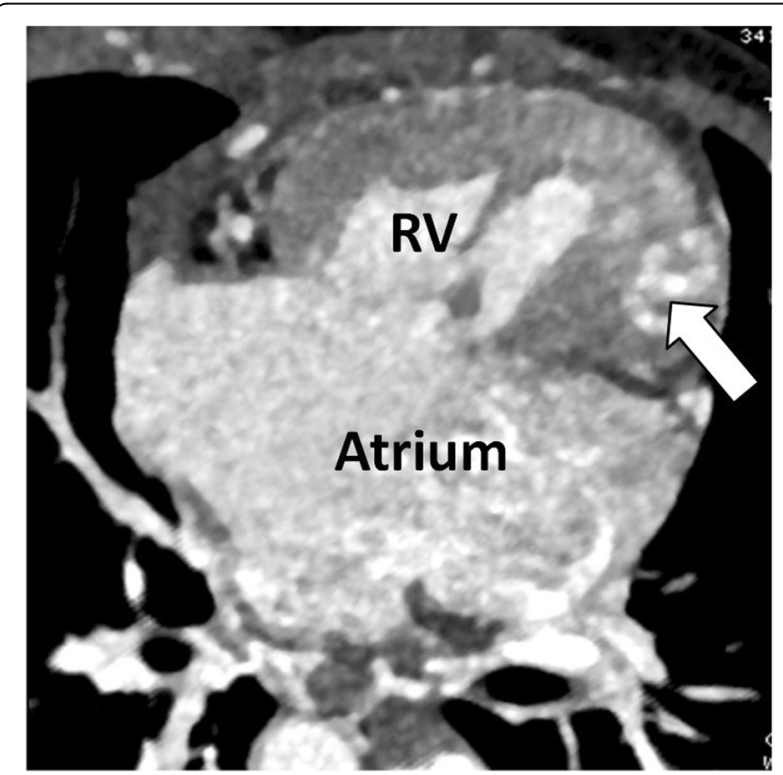

Fig. 1 Cardiac CT of a 2 days old infant showing single atrium (Atrium), univentricular right ventricle (RV) and rudimentary left ventricle (arrow) 


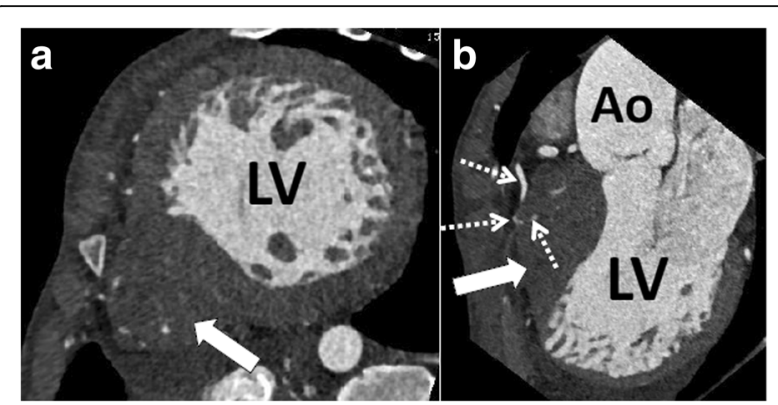

Fig. 2 Cardiac CT of a 1 month old boy showing univentricular left ventricle (LV) and rudimentary right ventricle (a and $\mathbf{b}$; arrows). Coronary artery fistula from right coronary artery draining into the rudimentary right ventricle was found (b; dashed arrows)

\section{Discussion}

$\mathrm{CT}$ is a robust alternative diagnostic modality for diagnosis of functional univentricular heart disease. In a significant number of patients in our cohort who had previous echocardiography, cardiac CT was able to provide additional information on extracardiac findings such as hypoplastic aortic arch, infracardiac type total anomalous pulmonary venous returns and hypoplastic left lung. For highly select indications, the risk profile may sometimes be in favour of using cardiac CT compared with other diagnostic methods when risks from anaesthesia are considered. Univentricular heart patients are exposed to relatively high cumulative radiation levels during staged palliation $[15,16]$. A single institution reports a median cumulative effective radiation dose of $25.7 \mathrm{mSv}$ from birth to 33 months of age, of which $78 \%$ was from catheterization [15]. Another study of cumulative radiation dose for patients with all forms of congenital

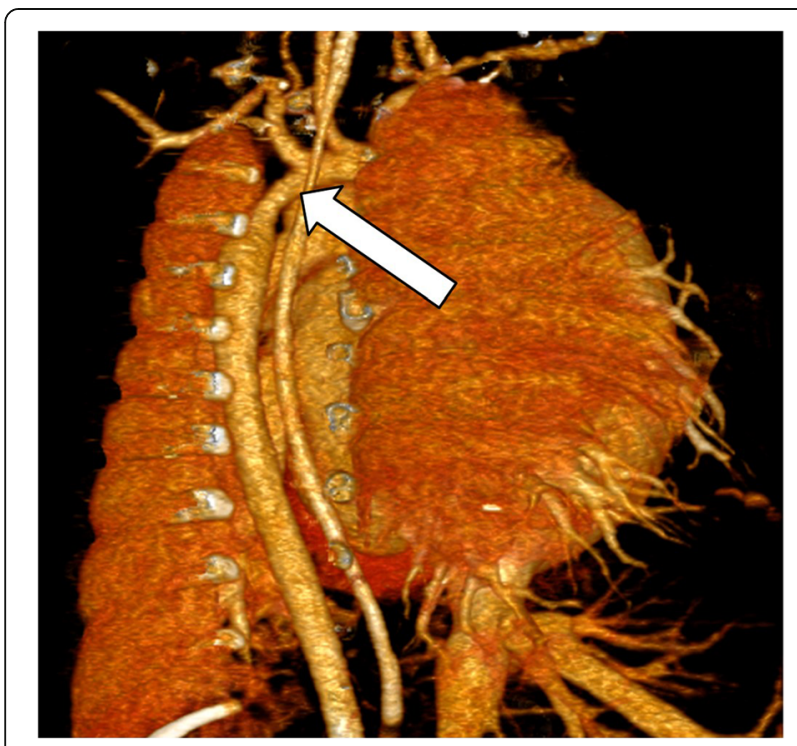

Fig. 3 Cardiac CT of a 1 month old univentricular right ventricle patient showing hypoplastic aortic arch (arrow)

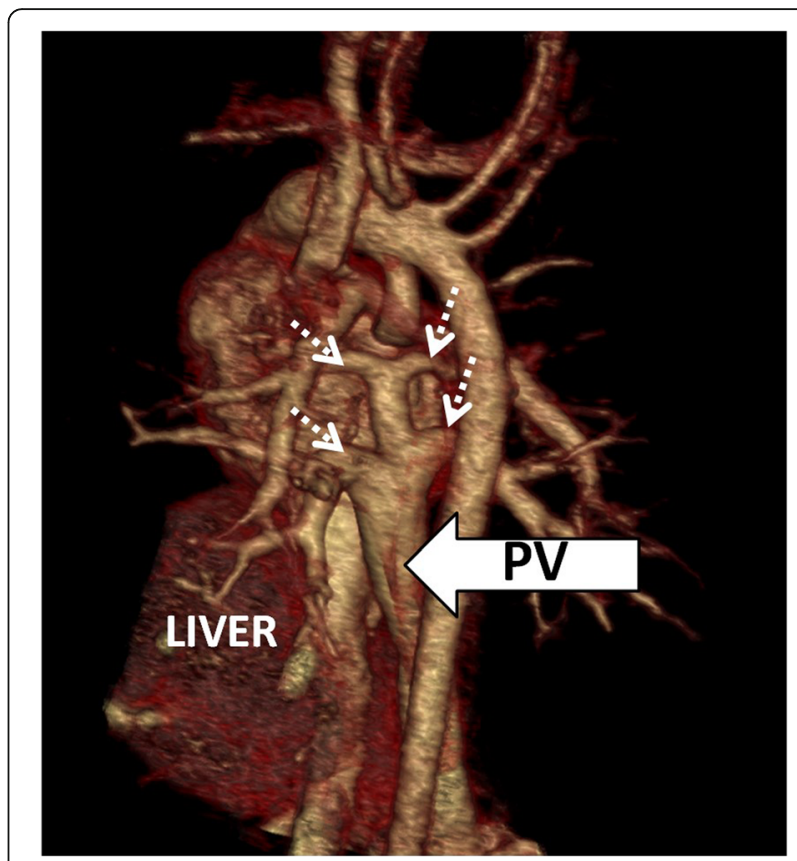

Fig. 4 Cardiac $\subset$ of a 1 year old univentricular left ventricle patient showing infracardiac type total anomalous pulmonary venous returns (dashed arrows) which draining into portal vein (arrow). (PV; portal vein)

heart disease showed that $5.3 \%$ of patients received over $20 \mathrm{mSv} /$ year with a median follow-up time of 4.3 years [17].

A recent study directly comparing radiation doses from diagnostic catheterization $(n=50$ cases) and computed tomography angiography $(n=50$ cases $)$ in children with congenital heart disease has shown 15-fold less radiation from CT angiography, although this was not specific to patients with single-ventricle heart disease [18]. Other studies using older CT scanner showing doses for CT angiography $(n=21)$ that are twofold higher than those in diagnostic cardiac catheterization $(n=117)$ [18]. These results show that the radiation dose from CT varies considerably depending on the type of scanner used and the aggressiveness of dose reduction. The image quality necessary for evaluation of coronary lesions in adult patients is rarely required for congenital applications, and patient-specific dose reduction must be implemented if a diagnostic strategy utilising $\mathrm{CT}$ is to be implemented. The standard diagnostic protocol at a majority of centers remains invasive catheterization before surgical palliation, despite data showing a favorable risk profile for non-invasive evaluation [3]. Non-invasive assessment before surgical palliation has shown similar operative outcomes compared with invasive catheterization involving lower risk as measured by radiation exposure, vascular access complications, length of anesthesia, and adverse events [19-21].

Our practice now uses CT preferentially for evaluation of anatomy before surgical palliation. Catheterization is 
preserved for patients in whom intervention is likely considered on the basis of echocardiography or clinical examination, and for patients with poor ventricular function and severe valve regurgitation in whom hemodynamics are considered relevant to clinical management. Some experts now propose a non-invasive algorythm for evaluation before surgical palliation in patients with single-ventricle heart disease considered to be at a low risk for requiring intervention [22-24].

Cardiovascular MRI is the most commonly used noninvasive advanced imaging modality in congenital heart disease but deep sedation or general anesthesia is required in young children, scan times are relatively long, and gadolinium is used in many patients for angiography. Anesthesia poses increased risk for patients with complex congenital heart disease undergoing MRI evaluation, and there is concern that repeated anesthesia exposure of young patients may have adverse neurological effects [25-34]. Gadolinium deposits have been found in brain tissue after repeated dosing in both children and adult patients, the significance of which is not yet known [35-38]. Risk assessment of non-invasive modalities should include assessment of risk from anesthesia and iodinated or gadolinium-based contrast exposure in addition to radiation exposure and vascular-access requirements.

Cardiac CT is an alternative imaging modality that can be used as part of the non-invasive imaging modality when cardiac MRI is considered to pose a high risk or when there is an imaging artifact $[39,40]$. When anesthesia is needed, a single breath-hold is required for data acquisition and the length of anesthesia will be relatively short.

Despite promising initial results, our study has potential limitations. The findings from the present study are retrospective and limited to single-center experience, the generalizability of the present results is limited. The present result is also limited by the number of patients, therefore, the interpretation of sensitivity may be limited.

\section{Conclusion}

Cardiac CT can diagnose accurately and be performed with a low radiation exposure in patients with the functional univentricular heart disease. The aorta, pulmonary artery and lung can be evaluated completely and simultaneously as well. Cardiac CT is an effective advanced noninvasive imaging modality to comprehensive evaluation the functional univentricular heart patients, particularly if cardiac MRI poses a high risk or is contraindicated.

\section{Abbreviations}

CCT: Cardiac computed tomography; CHD: Congenital heart disease; CMR: Cardiac magnetic resonance imaging; CT: Computed tomography; MAPCAs: Major aortopulmonary collateral arteries; mGy: Milligray; MIP: Maximum intensity projection; MRI: Magnetic resonance imaging; mSv: Millisievert; VRT: Volume-rendering technique

\section{Acknowledgements}

The authors would like to thank the Faculty of Medicine for its support.

Funding

This study has not been funded by any research grant.

Availability of data and materials

Statistical analysis of data is included in published tables. Please contact author for data requests.

\section{Authors' contributions}

NC and PM participated in design of the study. NC collected data throughout the study with analysis and interpretation of data completed by PM assisted with the recruitment of all patients from clinics. All authors read and approved the final manuscript and gave consent for publication.

\section{Ethics approval and consent to participate}

The study was conducted under institutional approval of the IRB of the Khon Kaen University, where this study was solely conducted. The IRB protocol number of this study is 800001189 . All participants gave individual consent to participation and for the use of all data collected during the study.

\section{Competing interests}

The authors declare that they have no competing interests.

\section{Publisher's Note}

Springer Nature remains neutral with regard to jurisdictional claims in published maps and institutional affiliations.

\section{Author details}

${ }^{1}$ Radiology Department, Faculty of Medicine, Khon Kaen University, Khon Kaen 40000, Thailand. ${ }^{2}$ Cardiology Unit, Internal Medicine Department, Faculty of Medicine, Khon Kaen University, Khon Kaen, Thailand.

Received: 8 November 2017 Accepted: 9 May 2018

Published online: 16 May 2018

\section{References}

1. Wilkinson $J$, Anderson RH. Anatomy of functionally single ventricle. World J Pediatr Congenit Heart Surg. 2012;3(2):159-64. https://doi.org/10.1177/ 2150135111421508. indexed in Pubmed: 23804770

2. Jacobs ML, Mayer JE. Congenital heart surgery nomenclature and database project: single ventricle. Ann Thorac Surg. 2000;69(4 Suppl):S197-204. indexed in Pubmed: 10798438

3. Feinstein JA, Benson DW, Dubin AM, Cohen MS, Maxey DM, Mahle WT, et al. Hypoplastic left heart syndrome: current considerations and expectations. J Am Coll Cardiol. 2012:59(1 Suppl):S1-42. https://doi.org/10. 1016/j.jacc.2011.09.022. indexed in Pubmed:22192720

4. Banka P, McElhinney DB, Bacha EA, et al. What is the clinical utility of routine cardiac catheterization before a Fontan operation? Pediatr Cardiol. 2010;31:977-85. https://doi.org/10.1007/s-246-010-973603. indexed in Pubmed:20503042

5. Stern KW, McElhinney DB, Gauvreau K, Geva T, Brown DW. Echocardiographic evaluation before bidirectional Glenn operation in functional single-ventricle heart disease: comparison to catheter angiography. Circ Cardiovasc Imaging. 2011:4:498-505. https://doi.org/10. 1161/CIRCIMAGING.110.963280. indexed in Pubmed:2219272

6. Garg R, Powell AJ, Sena L, Marshall AC, Geva T. Effects of metallic implants on magnetic resonance imaging evaluation of Fontan palliation. Am J Cardiol. 2005;95:688-91. https://doi.org/10.1016/j.amjcard.2004.10.053. indexed in Pubmed:15721124

7. Cronin EM, Mahon N, Wilkoff BL. MRI in patients with cardiac implantable electronic devices. Expert Rev Med Devices. 2012;9:139-46. https://doi.org/ 10.1586/erd.11.73. indexed in Pubmed:22404775

8. Ramalho J, Semelka RC, Ramalho M, Nunes RH, AlObaidy M, Castillo M. Gadolinium-based contrast agent accumulation and toxicity: an update. AJNR Am J Neuroradiol. 2016;37:1192-8. https://doi.org/10.3174/ajnr.A4615. indexed in Pubmed:26659341

9. Kanda T, Oba H, Toyoda K, Kitajima K, Furui S. Brain gadolinium deposition after administration of gadolinium-based contrast agents. Jpn J Radiol. 2016; 
34:3-9. https://doi.org/10.1007/s11604-015-0503-5. indexed in Pubmed: 26608061

10. Kanda T, Matsuda M, Oba H, Toyoda K, Furui S. Gadolinium deposition after contrast-enhanced MR imaging. Radiology. 2015;277:924-5. https://doi.org/ 10.1148/radiol.15150025. indexed in Pubmed:25742194

11. Han BK, Rigsby CK, Hlavacek A, et al. Computed tomography imaging in patients with congenital heart disease part I: rationale and utility. An expert consensus document of the Society of Cardiovascular Computed Tomography (SCCT): endorsed by the Society of Pediatric Radiology (SPR) and the North American Society of Cardiac Imaging (NASCI). J Cardiovasc Comput Tomogr. 2015;9:475-92. https://doi.org/10.1016/j.jcct.2015.07.004. indexed in Pubmed:26272851

12. Halliburton SS, Abbara S, Chen MY, et al. SCCT guidelines on radiation dose and dose-optimization strategies in cardiovascular CT. J Cardiovasc Comput Tomogr. 2011;5:198-224. https://doi.org/10.1016/j.jcct.2011.06.001. indexed in Pubmed:21723512

13. AAPM Task Group 23 of the Diagnostic Imaging Council CT Committee. The measurement, reporting, and Management of Radiation Dose in CT, AAPM report 96. College Park: American Association of Physicists in Medicine; 2008. p. 1-25. ISBN: 978-1-888340-73-0

14. AAPM Task Group 204. Size-Specific Dose Estimates (SSDE) in pediatric and adult body CT examinations, AAPM report no.204; 2011. p. 1-23. ISBN: 9781-936366-08-8

15. Downing TE, McDonnell A, Zhu X, et al. Cumulative medical radiation exposure throughout staged palliation of single ventricle congenital heart disease. Pediatr Cardiol. 2015;36:190-5. https://doi.org/10.1007/s00246-0140984-5. indexed in Pubmed:25096904

16. Johnson JN, Hornik CP, Li JS, et al. Cumulative radiation exposure and cancer risk estimation in children with heart disease. Circulation. 2014;130: 161-7. https://doi.org/10.1161/CIRCULATIONAHA.113.005425. indexed in Pubmed:24914037

17. Glatz AC, Purrington KS, Klinger A, King L, Huda W, Hlavacek AM. Cumulative exposure to medical radiation for children requiring surgery for congenital heart disease. J Pediatr. 2014;164:789-94; e10. https://doi.org/10. 1016/j.jpeds.2013.10.074. indexed in Pubmed:24321535

18. Watson TG, Mah E, Schoepf UJ, King L, Huda W, Hlavacek AM. Effective radiation dose in computed tomographic angiography of the chest and diagnostic cardiac catheterization in pediatric patients. Pediatr Cardiol. 2013;34:518-24. https:/doi. org/10.1007/s00247-009-1436-X. indexed in Pubmed:19997730

19. Brown DW, Gauvreau K, Powell AJ, et al. Cardiac magnetic resonance versus routine cardiac catheterization before bidirectional glenn anastomosis in infants with functional single ventricle: a prospective randomized trial. Circulation. 2007;116:2718-25. https://doi.org/10.1161/CIRCULATIONAHA.107. 723213. indexed in Pubmed:18025538

20. Brown DW, Gauvreau K, Powell AJ, et al. Cardiac magnetic resonance versus routine cardiac catheterization before bidirectional Glenn anastomosis: long-term follow-up of a prospective randomized trial. J Thorac Cardiovasc Surg. 2013;146:1172-8. https://doi.org/10.1016/j.jtcvs.2012.12.079. indexed in Pubmed:23380513

21. Han BK, Vezmar M, Lesser JR, et al. Selective use of cardiac computed tomography angiography: an alternative diagnostic modality before secondstage single ventricle palliation. J Thorac Cardiovasc Surg. 2014;148:1548-54. https://doi.org/10.1016/j.jtcvs.2014.04.047. indexed in Pubmed:24914037

22. Fogel MA. Is routine cardiac catheterization necessary in the management of patients with single ventricles across staged Fontan reconstruction? No! Pediatr Cardiol. 2005;26:154-8. https://doi.org/10.1007/s00246-004-0960-6. indexed in Pubmed:15868320

23. Fogel MA, Pawlowski TW, Whitehead KK, et al. Cardiac magnetic resonance and the need for routine cardiac catheterization in single ventricle patients prior to Fontan: a comparison of 3 groups: pre-Fontan CMR versus cath evaluation. J Am Coll Cardiol. 2012;60:1094-102. https://doi.org/10.1016/j. jacc.2012.06.021. indexed in Pubmed:4395971

24. Prakash A, Khan MA, Hardy R, Torres AJ, Chen JM, Gersony WM. A new diagnostic algorithm for assessment of patients with single ventricle before a Fontan operation. J Thorac Cardiovasc Surg. 2009;138:917-23. https://doi. org/10.1016/j.jtcvs.2009.03.022. indexed in Pubmed:19660367

25. Ramamoorthy C, Haberkern CM, Bhananker SM, et al. Anesthesia related cardiac arrest in children with heart disease: data from the Pediatric Perioperative Cardiac Arrest (POCA) registry. Anesth Analg. 2010;110: 1376-82. https://doi.org/10.1213/ANE.0b013e3181c9f927. indexed in Pubmed:20103543
26. Girshin M, Shapiro V, Rhee A, Ginsberg S, Inchiosa MA Jr. Increased risk of general anesthesia for high-risk patients undergoing magnetic resonance imaging. J Comput Assist Tomogr. 2009;33(2):312-5. https://doi.org/10.1097/ RCT.0b013e31818474b8. indexed in Pubmed:19346867

27. Dorfman AL, Odegard KC, Powell AJ, Laussen PC, Geva T. Risk factors for adverse events during cardiovascular magnetic resonance in congenital heart disease. J Cardiovasc Magn Reson. 2007;9:793-8. https://doi.org/10. 1080/10976640701545305. indexed in Pubmed:17891617

28. Rappaport B, Mellon RD, Simone A, Woodcock J. Defining safe use of anesthesia in children. N Engl J Med. 2011;364:1387-90. https://doi.org/10. 1056/NEJMp1102155. indexed in Pubmed:21388302

29. Hays SR, Deshpande JK. Newly postulated neurodevelopmental risks of pediatric anesthesia. Curr Neurol Neurosci Rep. 2011;11:205-10. https://doi. org/10.1016/.j.juro.2012.11.090. indexed in Pubmed:23178900

30. Wilder RT, Flick RP, Sprung J, et al. Early exposure to anesthesia and learning disabilities in a population-based birth cohort. Anesthesiology. 2009;110: 796-804. https://doi.org/10.1097/01.anes.0000344728.34332.5d. indexed in Pubmed:19293700

31. Flick RP, Katusic SK, Colligan RC, et al. Cognitive and behavioral outcomes after early exposure to anesthesia and surgery. Pediatrics. 2011;128:1053-61. https://doi.org/10.1542/peds.2011-0351. indexed in Pubmed:21969289

32. DiMaggio C, Sun LS, Kakavouli A, Byrne MW, Li G. A retrospective cohort study of the association of anesthesia and hernia repair surgery with behavioral and developmental disorders in young children. J Neurosurg Anesthesiol. 2009;21:286-91. https://doi.org/10.1097/ANA.0b013e3181a71f11. indexed in Pubmed:19955889

33. Fogel MA, Weinberg PM, Parave E, et al. Deep sedation for cardiac magnetic resonance imaging: a comparison with cardiac anesthesia. J Pediatr. 2008;152: 534-9. https://doi.org/10.1016/.jpeds.2007.08.045. indexed in Pubmed:18346511

34. Odegard KC, DiNardo JA, Kussman BD, et al. The frequency of anesthesiarelated cardiac arrests in patients with congenital heart disease undergoing cardiac surgery. Anesth Analg. 2007;105:335-43. https://doi.org/10.1213/01. ane.0000268498.68620.39. indexed in Pubmed:17646487

35. Miller JH, Hu HH, Pokorney A, Cornejo P, Towbin R. MRI brain signal intensity changes of a child during the course of 35 gadolinium contrast examinations. Pediatrics. 2015;136:1637-40. https://doi.org/10.1542/peds. 2015-2222. indexed in Pubmed:26574593

36. Kanda T, Oba H, Toyoda K, Furui S. Recent advances in understanding gadolinium retention in the brain. AJNR Am J Neuroradiol. 2016;37:E1-2. https://doi.org/10.3174/ajnr.A4586. indexed in Pubmed:26494697

37. Roberts DR, Holden KR. Progressive increase of $\mathrm{T} 1$ signal intensity in the dentate nucleus and globus pallidus on unenhanced T1-weighted MR images in the pediatric brain exposed to multiple doses of gadolinium contrast. Brain and Development. 2016;38:331-6. https://doi.org/10.1016/j. braindev.2015.08.009. indexed in Pubmed:26345358

38. Kanda T, Fukusato T, Matsuda M, et al. Gadolinium-based contrast agent accumulates in the brain even in subjects without severe renal dysfunction: evaluation of autopsy brain specimens with inductively coupled plasma mass spectroscopy. Radiology. 2015;276:228-32. https://doi.org/10.1148/ radiol.2015142690. indexed in Pubmed:25942417

39. Han BK, Lesser JR. CT imaging in congenital heart disease: an approach to imaging and interpreting complex lesions after surgical intervention for tetralogy of Fallot, transposition of the great arteries, and single ventricle heart disease. J Cardiovasc Comput Tomogr. 2013;7:338-53. https://doi.org/ 10.1016/j.jcct.2013.10.003. indexed in Pubmed:24331929

40. Khairy P, Van Hare GF, Balaji S, et al. PACES/HRS expert consensus statement on the recognition and management of arrhythmias in adult congenital heart disease. Heart Rhythm. 2014;30(10):1-63. https://doi.org/10.1016/j.cjca. 2014.09.002. indexed in Pubmed:25262867 